\title{
Twist in Chiral Interaction between Biological Helices
}

\author{
A. A. Kornyshev \\ Research Center "Jülich," D-52425 Jülich, Germany \\ and Institute for Theoretical Physics, University of California at Santa Barbara, Santa Barbara, California 93106 \\ S. Leikin* \\ Laboratory of Physical and Structural Biology, National Institute of Child Health \\ and Human Development, National Institutes of Health, Bethesda, Maryland 20892
}

(Received 3 August 1999)

\begin{abstract}
Using an exact solution for the pair interaction potential, we show that long, rigid, chiral molecules with helical surface charge patterns have a preferential interaxial angle $\sim \sqrt{R H} / L$, where $L$ is the length of the molecules, $R$ is the closest distance between their axes, and $H$ is the helical pitch. Estimates based on this formula suggest a solution for the puzzle of small interaxial angles in $\alpha$-helix bundles and in cholesteric phases of DNA.
\end{abstract}

PACS numbers: $87.15 .-\mathrm{v}$

The existence of all living things depends on the molecular chirality of helices inside them. Chiral amino acids form chiral $\alpha$ helices that self-assemble into structural domains of many proteins. Chiral DNA forms cholesteric liquid crystals right inside living cells [1].

Chiral interactions present many puzzles [2]. In the cholesteric phase (Fig. 2 below), DNA molecules rotate by a fraction of a degree from layer to layer [1]. Their interaxial angle is much smaller than expected [3], and so is the angle between long $\alpha$ helices in proteins. The corresponding cholesteric pitch is $\sim 0.4-5 \mu \mathrm{m}$ in DNA [1] and even larger in cholesteric assemblies of $\alpha$ helices in organic solvents [4]. One of the challenges of the physics of chiral macromolecules is to understand how this is encoded in intermolecular interactions [3].

In this paper, we suggest an explanation for the small angle puzzle. We start from a heuristic model of interaction between two long, net-neutral helices in an electrolyte-free environment (Fig. 1). We present qualitative arguments followed by rigorous derivations based on an exact solution for the interaction potential. We apply the model to $\alpha$-helix bundles in proteins and address the origin of the cholesteric pitch in DNA, extending our ideas to multimolecular interaction in an electrolyte solution.

Balance of forces. - Consider two identical, righthanded, rigid, net-neutral helices (Fig. 1) immersed in an electrolyte-free medium and forming a small interaxial angle $\psi(|\sin \psi| \ll 1)$. The length of each molecule $L$ is much larger than its helical pitch $H$.

Each helix produces an electric field that decays as $\exp (-g r)$ with the distance $r$ away from it $(g=2 \pi / H)$ [5]. The fields overlap over the length $L_{\text {eff }}(\psi)$ which rapidly varies with $\psi$ and diverges at $\psi \rightarrow 0$ for infinite helices. The energy of interaction between helices is $E_{\text {int }}=u(\psi) L_{\text {eff }}$. The linear energy density, $u(\psi)$, does not diverge at $\psi \rightarrow 0$. It can be expanded at small $\psi$ so that

$$
E_{\mathrm{int}}=\left[u_{0}(R, g)+u_{1}(R, g) \psi+\ldots\right] L_{\mathrm{eff}}(\psi) .
$$

Here $R$ is the closest approach distance between the molecular axes, and $u_{1}(R, g) \psi$ is the energy density of chiral interaction that defines the direction of favorable twist [6].

The helices experience two torques, the "overlap torque" $\left(t_{1}=-u\left[d L_{\text {eff }} / d \psi\right]\right)$ and the "chiral torque" $\left(t_{2}=\right.$ $\left.-[d u / d \psi] L_{\text {eff }}\right)$. If the helices are free to rotate around their axes, they will always select the most favorable alignment at which $u<0$ and the molecules attract each

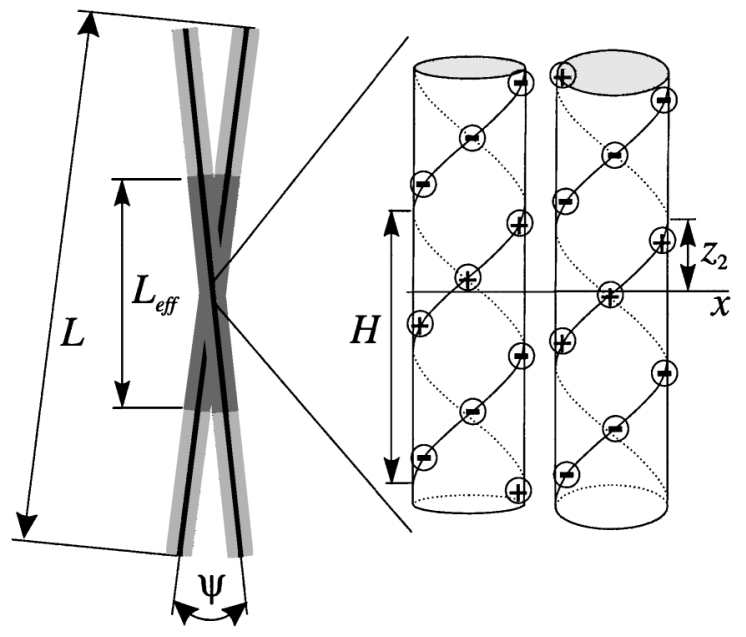

FIG. 1. Interaction between two long net-neutral helical macromolecules in a nonpolar environment. Left: The molecules are shown by solid lines, their electric fields by light gray shading, and the overlap (interaction) area by dark gray shading. Right: A small fragment of the overlap area is magnified in a side view. We assume that the surface charge pattern is composed of one negatively charged and one positively charged thin spiral lines shifted with respect to each other by approximately one-half of the pitch. To describe the alignment of each helix $(\nu=1,2)$, we use the axial distance $z_{\nu}$ between the positively charged strand and the $x$ axis connecting the points of the closest approach between molecules (as shown for molecule 2 on the right). At $z_{1}=z_{2}$, the positively charged strand of one molecule opposes the negatively charged strand of the other molecule, creating a strong attraction. We assume that the molecules cross each other approximately in the middle. 
other (Fig. 1). Then, the overlap torque tends to reduce $|\psi|$ to maximize the attraction. However, the chiral torque tends to increase $|\psi|$. The competition between these torques establishes a nonzero angle in equilibrium.

At small $\psi$, there are two distinct regions with different behavior of $L_{\mathrm{eff}}(\psi)$.

(1) $\left(|\psi|>\psi_{*}\right)$ : Tips of the helices are separated by more than $g^{-1}$. They contribute little to the interaction and $L_{\text {eff }} \propto 1 /(g|\sin \psi|)$ (Fig. 1), i.e.,

$$
L_{\mathrm{eff}}(\psi)=\frac{\gamma(R g)}{g|\sin \psi|} .
$$

The coefficient $\gamma(R g)$ is derived below [see Eq. (10)]. Within this region, $t_{1} / t_{2} \approx\left(u_{0} / u_{1}|\psi|\right) \gg 1$ since $|\psi| \ll$ 1. The overlap torque wins and it reduces $|\psi|$.
(2). $\left(|\psi| \ll \psi_{*}\right)$ : Tips of the helices overlap and $L_{\text {eff }}$ stops following Eq. (2). It levels off and has a maximum $L_{\text {eff }}=L$ at $\psi=0$ so that $\left(d L_{\text {eff }} / d \psi\right)_{\psi \rightarrow 0} \rightarrow 0$ and $t_{1} / t_{2} \ll 1$. The chiral torque wins in this region.

The torques become equal at the crossover from the first to the second region. Exact evaluation of the equilibrium interaxial angle requires the exact $L_{\text {eff }}(\psi)$ in the crossover range. However, we can estimate that the crossover occurs at $|\psi| \sim \psi_{*}$, where

$$
\psi_{*}=\frac{\gamma(R g)}{L g}
$$

is obtained by extrapolating Eq. (2) to $L_{\text {eff }}(\psi)=L$. The value of $\psi_{*}$ may serve as an upper estimate for the interaxial angle. According to Eq. (3), this angle is small for long molecules.

Rigorous derivation. - The charge pattern on each molecule shown in Fig. 1 can be described in its own cylindrical coordinate frame with the long axis of the helix as the $z$ axis. The Fourier transform of the charge density in the molecular frame is given by

$$
\tilde{\sigma}_{\nu}(q, n)=\frac{Z e_{0} g}{2 \pi a}\left[1-(-1)^{n}\right] \exp \left(i n g z_{\nu}\right) \delta(q+n g),
$$

where $\tilde{\sigma}_{\nu}(q, n)=(2 \pi)^{-1} \int_{0}^{2 \pi} d \phi \int_{-\infty}^{\infty} d z \sigma_{\nu}(z, \phi) e^{i n \phi} e^{i q z}, \nu(=1,2)$ labels the molecules, $e_{0}$ is the elementary charge, $Z$ is the number of elementary charges per helical pitch on each strand, and $z_{\nu}$ defines the alignment of each helix (Fig. 1). After substitution of Eq. (4), into the expression for $E_{\text {int }}$ derived in [6], we find

$$
\begin{aligned}
\frac{E_{\text {int }}(\psi \neq 0)}{k_{B} T}=-\frac{2 Z^{2} l_{B} g}{\pi|\sin \psi|} \sum_{\substack{i, j=-\infty \\
n=2 i+1 \\
m=2 j+1}}^{\infty} \frac{\cos \left[n g z_{1}-m g z_{2}\right]}{|m| \sqrt{1+\tilde{w}_{n, m}^{2}(\psi)}} I_{n}(|n| g a) I_{m}(|m| g a) e^{-|m| R g \sqrt{1+\tilde{w}_{n, m}^{2}(\psi)}} \\
\times\left[\sqrt{1+\tilde{w}_{m, n}^{2}(\psi)}+\tilde{w}_{m, n}(\psi)\right]^{n}\left[\sqrt{1+\tilde{w}_{n, m}^{2}(\psi)}+\tilde{w}_{n, m}(\psi)\right]^{m},
\end{aligned}
$$

where $a$ is the radius of the helices, $\varepsilon$ is the dielectric constant of the medium, $l_{B}=e_{0}^{2} / \varepsilon k_{B} T$ is the Bjerrum length, and

$$
\tilde{w}_{n, m}(\psi)=\frac{n g-m g \cos \psi}{|m g| \sin \psi} .
$$

Equation (5) is valid at all $\psi \neq 0$, when the molecules are long enough so that their tips do not contribute much to the interaction. At $\psi=0$ (see Ref. [6]),

$$
\frac{E_{\mathrm{int}}(\psi=0)}{k_{B} T}=-\frac{4 Z^{2}}{\pi^{2}} L l_{B} g^{2} \sum_{\substack{i=0 \\ n=2 i+1}}^{\infty} \cos \left[n g\left(z_{1}-z_{2}\right)\right]\left[I_{n}(n g a)\right]^{2} K_{0}(n R g) .
$$

In Eqs. (5) and (7), $I_{n}(x)$ and $K_{0}(x)$ are modified Bessel functions. At $L \rightarrow \infty$, Eqs. (5) and (7) are exact. They contain no approximations or assumptions, except for the choice of the surface charge distribution [Eq. (4)].

The summation in Eqs. (5) and (7) can be truncated after $n, m= \pm 1$, because typically $R g \gg 1$ [7]. Thus,

$$
\begin{aligned}
\frac{E_{\mathrm{int}}(\psi \neq 0)}{k_{B} T} \approx-\frac{4 Z^{2} l_{B} g\left[I_{1}(g a)\right]^{2}}{\pi|\sin \psi|}\left\{\frac{e^{-\frac{R_{g}}{|\cos (\psi / 2)|}}}{|\cos (\psi / 2)|}\left(1+\frac{\sin \psi}{2|\cos (\psi / 2)|}\right)^{2} \cos \left[g\left(z_{1}-z_{2}\right)\right]\right. \\
\left.+\frac{e^{-\frac{R g}{|\sin (\psi / 2)|}}}{|\sin (\psi / 2)|}\left(1-\frac{\sin \psi}{2|\sin (\psi / 2)|}\right)^{2} \cos \left[g\left(z_{1}+z_{2}\right)\right]\right\} .
\end{aligned}
$$

By plotting $E_{\text {int }}$, one can easily find that the energy has a minimum at $z_{1}=z_{2}$ and $\psi \rightarrow 0$ [8].

Small angle behavior.-Expanding $E_{\text {int }}(\psi \neq 0)$ in small $\psi$ at $z_{1}=z_{2}$ and comparing the result with Eq. (7), we recover Eqs. (1) and (2), where

$$
\frac{u_{0}(R, g)}{k_{B} T}=\frac{u_{1}(R, g)}{k_{B} T}=-\frac{4 Z^{2}}{\pi^{2}} l_{B} g^{2}\left[I_{1}(g a)\right]^{2} K_{0}(R g),
$$

and

$$
\gamma(R g)=\frac{\pi e^{-R g}}{K_{0}(R g)}
$$

Using $R g \gg$, we find from Eqs. (3) and (10) that

$$
\psi_{*} \approx \frac{\sqrt{2 \pi R / g}}{L}=\frac{\sqrt{R H}}{L},
$$


which is a remarkably simple combination of the only three length scales in the system. The energy gain upon the twist from $\psi=0$ to $\psi \sim \psi_{*}$ is

$$
\frac{E_{*}}{k_{B} T} \sim \frac{u_{1}(R, g) \psi_{*} L}{k_{B} T} \approx \frac{2 Z^{2} l_{B}}{\pi^{2} a} e^{-g(R-2 a)},
$$

where we also assumed that $g a \gg 1$.

Interaction between $\alpha$ helices in proteins. - Many proteins incorporate bundles of $\alpha$ helices. The backbone of each $\alpha$ helix contains a spiral of negatively charged carbonyl oxygens and a shifted spiral of positively charged amide hydrogens. In terms of the charge distribution, it resembles the heuristic model analyzed above, where $a \approx$ $2.3 \AA, H \approx 5.5 \AA, L / H \approx 3-10, R \approx 7-12 \AA[9,10]$, $Z \approx 1.7\left(\sim 0.5 e_{0}\right.$ per carbonyl or amide, $\sim 3.5$ groups per helical turn), and $\varepsilon \approx 2\left(l_{B} \approx 300 \AA\right)$. For such helices, we find $\psi_{*} \sim 0.1-0.5 \mathrm{rad}\left(7^{\circ}-30^{\circ}\right)$.

Of course, amino acid side chains impose packing constraints that play a major role in determining the structure of $\alpha$-helix bundles in proteins [9]. Still, electrostatic interaction between backbones of $\alpha$ helices that do not have bulky side chains $(R \approx 7-8 \AA)$ is energetically significant $\left(E_{*} \sim 2-5 k_{B} T\right)$ and it may be an important player as well [11]. For instance, steric interactions define a set of preferential interaxial angles rather than a single angle [9]. Electrostatics may then determine which angle from the set is most favorable. It may not be a coincidence that the average observed angle $\left(\sim 19^{\circ}[9]\right)$ is in the middle of the range predicted for chiral electrostatic interactions.

Cholesteric pitch of DNA. - Concentrated solutions of 500- $\AA$-long DNA in 10-300 mM salt form a cholesteric phase (Fig. 2) at $32<R<49 \AA[12,13]$. Such DNA fragments are short enough to behave as rigid rods [14] and long enough to have many helical turns, $L / H \approx 15$.

Direct measurements of intermolecular forces demonstrated that the energetics of this cholesteric phase is determined primarily by electrostatic interactions [15]. The interactions are essentially pairwise since each DNA helix overlaps with only one molecule in the layer below it [16].
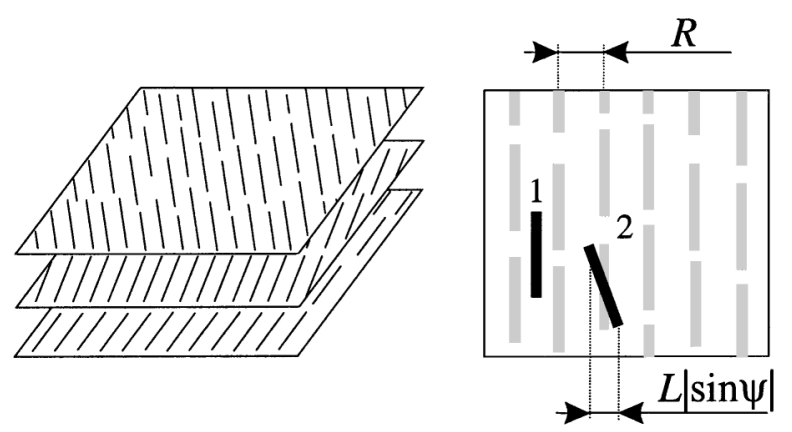

FIG. 2. Alignment of DNA helices in the cholesteric phase. Left: A sketch of the cholesteric phase that consists of layers of parallel molecules. Each layer is slightly rotated with respect to the layer underneath. Right: Top view of a molecular layer showing most favorable alignments of molecules in the layer above (black rods) (1) when molecules are homogeneously charged and (2) when molecules have helical patterns of fully balanced surface charges.
Furthermore, only nearest neighbor pairs contribute to the energy because of the rapid, exponential decay of the field.

The net interaction between each of the two molecules can be viewed as a sum of two forces. The first one is a repulsion due to the fraction of DNA charge not balanced by bound or condensed counterions ( $20 \%-25 \%$ of "naked" DNA charge [17]). This repulsion is the same as between homogeneously charged cylinders in an electrolyte solution [6]. It favors parallel $(\psi=0)$ alignment of helices in a multimolecular ensemble (Fig. 2) because this maximizes intermolecular separation and reduces the repulsion $[2,18]$.

The second force is due to the compensated part of DNA charge. At optimal molecular alignment, it is an attraction between negatively charged phosphate strands on one molecule and positively charged grooves on the opposing molecule [5,6,19]. The physics of this force is the same as in our heuristic model (Fig. 1), but some details are different. Specifically, DNA is a double-stranded helix and it has a more complicated surface charge pattern than in Fig. 1 [20]. In addition, electrolyte reduces the decay length of the electric field from $g^{-1}$ to $\left(g^{2}+\kappa_{D}^{2}\right)^{-1 / 2}$, where $\kappa_{D}^{-1}$ is the Debye length. These details are important for accurate quantitative predictions [6], but for order-of-magnitude estimates they can be neglected. From Eqs. (11) and (12), where $L \approx 500 \AA, a \approx 10 \AA, H \approx$ $34 \AA, R \sim 40 \AA,|Z| \approx 40[21]$, and $l_{B} \approx 7 \AA(\varepsilon \approx 80)$, we find $\psi_{*} \approx 0.07 \mathrm{rad}$ and $E_{*} \approx 2-5 k_{B} T$ [20].

The competition between the first force favoring $\psi=0$ and the second force favoring $|\psi| \sim \psi_{*}$ determines whether DNA will form a cholesteric phase and the pitch of this phase [22]. Even without a many-body statistical theory, it is clear that the equilibrium interaxial angle should be smaller than $\psi_{*}$ and, therefore, the expected pitch should be larger than $P_{*}=2 \pi R / \psi_{*} \approx 0.4 \mu \mathrm{m}$, as it is observed $[1,12,13]$. As it is also observed [12,13], the pitch should not depend much on the salt concentration at $10-300 \mathrm{mM}$ salt. Indeed, since $g \approx 0.2 \AA^{-1}, P_{*} \approx$ $\left(g^{2}+\kappa_{D}^{2}\right)^{1 / 4}(2 \pi R)^{1 / 2} L$ is almost unaffected by the corresponding variation of $\kappa_{D}$ from 0.03 to $0.2 \AA^{-1}$.

DNA molecules much longer than one persistence length also form a cholesteric phase with about the same pitch as $500 \AA$ fragments [1]. In this case, the theory is more complicated since our description of molecules as straight, rigid rods does not apply. However, assuming that each molecule behaves as a collection of independent, one-persistence-length-long fragments [14], we can explain the observations.

Thus, the macroscopic cholesteric pitch in DNA aggregates may have the following origin. When tips of one-persistence-length-long DNA fragments are separated (in lateral projection) by more than $g^{-1}$, the attraction between negatively charged phosphate strands and positively charged grooves tends to reduce $\psi$. When the tips are separated by $\geq \kappa_{D}^{-1}$, the repulsion associated with the uncompensated charge of DNA also tends to reduce $\psi$. These two forces overpower the chiral torque, reducing the separation of the tips and resulting in the pitch 
$\geq\left(g^{2}+\kappa_{D}^{2}\right)^{1 / 4}(2 \pi R)^{1 / 2} L \sim 8 L \sim 0.4 \mu \mathrm{m}$. A more detailed theory, based on the same ideas, also shows why the chiral torque may disappear at $R \leq 32 \AA$ (a possible solution for the puzzle of nematic-to-cholesteric transition at $R=32 \AA$ ) and it demonstrates that the direction of the chiral torque can be reversed upon a change in the counterion binding pattern or in separation [6].

In conclusion, let us emphasize that the goal of this Letter is to illustrate on a conceptual level why chiral helical molecules may not twist more than a couple degrees in the cholesteric phase of DNA and in bundles of long $\alpha$ helices. Of course, estimates are not a substitute for an accurate statistical theory that accounts for the pair potential between helices of finite length and for thermal motion. They are intended only to pave the way. The agreement with experiments indicates, however, that we may be on the right track.

We thank V. A. Parsegian and D. C. Rau for useful discussions. This work was supported by the NSF Grant No. PHY94-07194. In addition, A. A. K. acknowledges the financial support of his visits to Bethesda by the National Institute of Child Health and Human Development, $\mathrm{NIH}$, which allowed completion of this project.

*To whom reprint requests should be addressed; (Bldg. 12A, Rm. 2041, NIH, Bethesda, MD 20892).

Email address: leikin@helix.nih.gov

[1] Yu. M. Yevdokimov, S. G. Skurdin, and V. I. Salyanov, Liq. Cryst. 3, 1443 (1988); F. Livolant, Physica (Amsterdam) 176A, 117 (1991).

[2] P. G. deGennes and J. Prost, The Physics of Liquid Crystals (Clarendon Press, Oxford, 1993), 2nd ed.

[3] A. B. Harris, R. D. Kamien, and T. C. Lubensky, Phys. Rev. Lett. 78, 1476 (1997); Rev. Mod. Phys. 71, 1745 (1999).

[4] C. Robinson, Tetrahedron 13, 219 (1961); F. Livolant, J. Phys. (Paris) 47, 1605 (1986); D. B. Dupre and R.W. Duke, J. Chem. Phys. 63, 143 (1975).

[5] A. A. Kornyshev and S. Leikin, J. Chem. Phys. 107, 3656 (1997); 108, 7035(E) (1998); A. A. Kornyshev and S. Leikin, Proc. Natl. Acad. Sci. U.S.A. 95, 13579 (1998).

[6] A. A. Kornyshev and S. Leikin, preprint 9911360 at xxx.lanl.gov.

[7] The diameter ( $2 a)$ of most biological helices is comparable to their pitch $(2 a / H \sim 1)$. Since $R g \geq 2 a g=4 \pi a / H$, we find that $R g \geq 2 \pi$ and the approximation of $R g \gg 1$ is almost always valid.

[8] From Eq. (8), one can estimate the cost of rotation of each helix around its axis at fixed height. Such rotation of helix $\nu$ by $\Delta \phi_{\nu}$ results in the change of $z_{\nu}$ by $g z_{\nu}$. Both for $\alpha$ helices and for DNA the cost is much larger than $k_{B} T$ (except at the very edge of existence of the cholesteric phase of DNA, $R \approx 50 \AA$ ). Thus, in the first approximation, thermal rotation can be neglected.
[9] See, e.g., C. Chothia, M. Levitt, and D. Richardson, J. Mol. Biol. 145, 215 (1981); P. B. Harbury, P. S. Kim, and T. Alber, Nature (London) 371, 80 (1994).

[10] For heuristic reasons, we use this crude approximation of the $\alpha$-helix charge pattern. In principle, the formalism developed in [6] allows more precise analysis.

[11] The contribution of van der Waals forces to the chiral energy calculated based on the formulas derived by S. A. Issaenko, A. B. Harris, and T. C. Lubensky [Phys. Rev. E 60, 578 (1999)] gives $E_{*}^{\mathrm{vdW}} \approx 0.06 k_{B} T$ at $R=7 \AA$; i.e., it is much smaller than the contribution of electrostatic forces.

[12] A. Leforestier and F. Livolant, Biophys. J. 65, 56 (1993); D. H. Van Winkle, M. W. Davidson, W. X. Chen, and R. L. Rill, Macromolecules 23, 4140 (1990).

[13] D. Durand, J. Doucet, and F. Livolant, J. Phys. II (France) 2, 1769 (1992).

[14] The persistence length of DNA is $\sim 500-1000 \AA$, depending on the ionic strength.

[15] H. H. Strey, V. A. Parsegian, and R. P. Podgornik, Phys. Rev. E 59, 999 (1999).

[16] In the direction normal to the layer below, DNA traverses the distance $L|\sin \psi| \approx L \psi \leq 25 \AA$ (Fig. 2). This is less than the interaxial separation $32 \AA<R<49 \AA$ [13] between the molecules in this layer.

[17] G. S. Manning, Q. Rev. Biophys. 11, 179 (1978).

[18] This interaction tends to put axes of two isolated molecules at $\psi= \pm \pi / 2$ [S. L. Brenner and V. A. Parsegian, Biophys. J. 14, 327 (1974)]. However, in a multimolecular aggregate such alignment reduces the minimal distance between molecules at the same density of the phase (compared to hexagonal packing of parallel helices). As a result, this homogeneous repulsion favors $\psi=0$.

[19] A. A. Kornyshev and S. Leikin, Phys. Rev. Lett. 82, 4138 (1999).

[20] In the absence of counterion chemisorption, one should speak of an excess negative charge on phosphate strands and an excess positive charge in grooves separating the strands, rather than of a fixed pattern such as in Fig. 1. Quantitatively, this excess may vary with the counterion nature, but most monovalent counterions do exhibit a preference to reside in grooves [see, e.g., V.N. Bartenev, E. I. Golovanov, K. A. Kapitonova, M. A. Mokulskii, L. I. Volkova, and I. Ya. Skuratovskii, J. Mol. Biol. 169, 217 (1983); X. Shui, L. McFail-Isom, G. G. Hu, and L.D. Williams, Biochemistry 37, 8341 (1998)]. Details of the counterion pattern may have a strong effect on the absolute value of the energy of chiral interactions, but not on the most energetically favorable angle between helices [6].

[21] Two phosphate strands contribute 20 elementary charges per pitch. Their electrostatic images on the nonpolar dielectric core of DNA effectively double the charge to $Z \sim 40$.

[22] In 1:1 electrolytes, the first force is stronger than the second one, resulting in net repulsion between molecules [19]. However, the first force produces no torque at $\psi=0$ (as it follows from symmetry). As a result, the torque from the second force is dominant at $|\psi| \ll \psi_{*}$. 